\title{
Switching stiction and adhesion of a liquid on a solid
}

\author{
Stijn F. L. Mertens ${ }^{1,2 * \pi}$, Adrian Hemmi ${ }^{3 \uparrow}$, Stefan Muff ${ }^{3}$, \\ Oliver Gröning ${ }^{4}$, Steven De Feyter ${ }^{1}$, Jürg Osterwalder ${ }^{3}$, Thomas Greber ${ }^{3 *}$ \\ ${ }^{1}$ Department of Chemistry, KU Leuven, \\ Celestijnenlaan 200F, 3001 Leuven, Belgium \\ ${ }^{2}$ Institut für Angewandte Physik, Technische Universität Wien, \\ Wiedner Hauptstrasse 8-10/E134, 1040 Wien, Austria \\ ${ }^{3}$ Physik-Institut, Universität Zürich, \\ Winterthurerstrasse 190, 8057 Zürich, Switzerland \\ ${ }^{4}$ Empa, Swiss Federal Laboratories for Materials Science and Technology, \\ Überlandstrasse 129, 8600 Dübendorf, Switzerland \\ "These authors contributed equally
}

To whom correspondence should be addressed: stmerten@gmail.com, greber@physik.uzh.ch

When a gecko moves on a ceiling it makes use of adhesion and stiction. Stiction, also called static friction, is omnipresent in the microscopic and the macroscopic world and is related to adhesion and sliding friction [1]. Although important for most locomotion processes, the concepts of adhesion, stiction and sliding friction are often only empirically correlated. This urges a more detailed understanding, which will also improve today's ever-smaller devices such as micro- and nanoelectromechanical switches (MEMS/NEMS) [2]. Here, we show how stiction and adhesion are related for a liquid on a hexagonal boron nitride monolayer on rhodium [3], by measuring dynamic contact angles in two distinct states of the liquid-solid interface. Both stiction and adhesion can be reversibly switched between two pairs of values in applying different electrochemical potentials where atomic hydrogen is intercalated or not. We ascribe the change in adhesion to a change in lateral electric fields of in-plane $2 \mathrm{~nm}$ dipole rings [4], as it cannot be explained by the change in surface roughness known from the Wenzel 
model [5]. While the change in adhesion can be calculated for the present system [6], the stiction at a solid-liquid interface remains inaccessible for $a b$ initio methods. The inorganic $h$-BN/Rh hybrid is very stable and represents a new class of switchable surfaces with great application potential in the study of adhesion, friction and lubrication.

Every object at rest sticks with some adhesion to its substrate. If this object shall be moved, a force has to act on it. This force has to overcome the stiction threshold, above which the object starts to move and below which it sticks to the support. This fundamentally simple principle indicates a relation between adhesion and stiction that is valid for single atom manipulation [7] up to the everyday experience of moving ourselves. Stiction is also related to sliding friction [1,8] and to surface diffusion [9]. Empirically they are connected with dimensionless coefficients that relate e.g. the diffusion barrier with the adsorption energy or the sliding friction force with the load. The advent of single atom probes on surfaces and the measurement of the heat of adsorption have made the Evans-Polanyi relation, which postulates proportionality between the activation energy for diffusion and the adsorption energy experimentally accessible [10]. For macroscopic objects on the other hand, Amontons' first law stating sliding friction to be proportional to the load, is much more complex because of the many length scales involved. This complexity also applies to stiction and consequently, an ab initio understanding is difficult [11]. For such problems we rely on clear-cut model systems and methods to study the influence of microscopic effects on their macroscopic expression and vice versa. Experimentally this can be achieved with responsive surfaces.

In responsive surfaces, a collective change of a microscopic atomic or molecular parameter triggers a macroscopic property change such as the wetting angle of a liquid and such surfaces are at the core of nanoscience and smart materials research [12-14]. Typically, the responsiveness is invoked by a change of conformation or charge state of organic molecules at the solid-liquid interface, which can be triggered by light [12], temperature [13] or electric fields [14]. The organic molecules that are at the basis of the switching, however, render these surfaces quite fragile and limited to near-ambient temperatures. In contrast, the inorganic $h-\mathrm{BN} / \mathrm{Rh}$ hybrid in focus here has very high thermal and chemical stability (see Methods), and therefore signifies a new class of responsive surfaces with considerable technological promise. The stability of the $h-\mathrm{BN} / \mathrm{Rh}$ system is related to the covalently 
bonded network of the $h$-BN "skin" in comparison with the weaker supramolecular interactions in self-assembled monolayers.

The boron nitride nanomesh is a corrugated monolayer of $h$-BN on rhodium $[3,15]: 13 \times 13 h$ $\mathrm{BN}$ units form a superhoneycomb structure on $12 \times 12 \mathrm{Rh}(111)$ unit cells, where particularly strong lateral electric fields exist that are for instance decisive for the self-assembly of molecules [4]. The nanomesh structure is stable in vacuum up to $1000{ }^{\circ} \mathrm{C}$ and survives immersion into liquids $[4,16]$. In ultrahigh vacuum, it was found that the boron nitride layer could be made flat by intercalation of atomic hydrogen [17]. In this letter, we demonstrate this effect under electrochemical conditions and show $h$-BN/Rh(111) to be a surface with switchable wetting and adhesion.

The electrochemical switching of the surface texture is based on intercalation of atomic hydrogen, as is demonstrated in Figure 1. Figure 1a shows cyclic voltammograms for a clean $\mathrm{Rh}$ (111) film and a $h$-BN nanomesh sample in perchloric acid solution in a sessile drop configuration [18]. In the negative scan direction, the $\mathrm{Rh}(111)$ reference voltammogram exhibits the typical atomic hydrogen adsorption peak just before the onset of molecular hydrogen evolution [19]. On $h-\mathrm{BN} / \mathrm{Rh}(111)$, this peak occurs at less negative potentials, which suggests that the process is energetically slightly more favourable on the nanomesh. During the reverse scan, the single desorption peak on $\mathrm{Rh}(111)$ shows a double peak for $h$ $\mathrm{BN} / \mathrm{Rh}(111)$, which indicates a 2-stage desorption process. Integration of the hydrogen adsorption peak yields $75 \mu \mathrm{C} / \mathrm{cm}^{2}$, which amounts to one third of the bare $\mathrm{Rh}(111)$ measurement where one monolayer of hydrogen may be adsorbed, and confirms earlier observations [16,17]. On continued cycling (Figure 1b) a slight sharpening of the adsorption peak occurs; the charge related to the peak integral changes by less than $15 \%$, demonstrating the stability of the $h$-BN overlayer upon cycling.

The changes in nanotexture that accompany the hydrogen adsorption and desorption were visualised directly by in situ electrochemical scanning tunnelling microscopy (EC-STM). Figures $1 \mathrm{c}-\mathrm{f}$ show the same area for different substrate potentials. Initially, at potential $E_{1}$ (Figure 1c), the hexagonal pattern of pores separated by wires is observed [16, 17]. The 3.2 $\mathrm{nm}$ periodicity can also be seen in the autocorrelation of a given section from one terrace and the corrugation is reflected by the root mean square (RMS) roughness. Crossing of the hydrogen adsorption peak (potential $E_{2}$, Figure 1d) leads to flattening of the surface, which is 
reflected in the vanishing of the superstructure and a decrease of the RMS roughness. A tip change between Figure 1c and Figure 1e caused a contrast inversion of the pore, which does, however, not affect the RMS analysis. When the potential is set back to the initial value, the corrugation gradually reappears (Figure 1e), which indicates that deintercalation is slower than the forward process (see Methods and Extended Data Figure 2). After the cycle (Figure 1f), the superhoneycomb shows different imperfections (e.g., some pores appear connected). The physical picture that emerges is illustrated in Figure 1g: Electrochemical intercalation of hydrogen reversibly flattens the $\mathrm{h}-\mathrm{BN} / \mathrm{Rh}$ nanomesh, and in doing so switches off the dipole rings in the surface.

In order to prove unequivocally that hydrogen intercalation is at the basis of the observed switching effect, we conducted an ambient-to-vacuum transfer experiment with the goal to quantify the intercalated hydrogen. The electrochemical treatment was performed in heavy water, and the $h-\mathrm{BN} / \mathrm{Rh}(111)$ sample was extracted from the solution under potential control and transferred in less than 10 minutes to vacuum (see Methods) where thermal desorption spectroscopy (TDS) was carried out, as shown in Figure 2. Deuterium desorption from the sample held at potential $E_{2}$ (flat state) is obvious, whereas no significant desorption took place from a sample that was kept at $E_{1}$ (corrugated) throughout. The desorption temperature indicates slightly higher binding energy as compared to hydrogen on bare $\mathrm{Rh}(111)$ [20]. This further confirms hydrogen intercalation, since hydrogen (or deuterium) on top of $h$-BN would not bind as strongly as on rhodium and would not survive the transfer from the liquid to the vacuum. Our experiments do not show atomistic details of how the hydrogen intercalates, though it was claimed by $\mathrm{Hu}$ et al. [21] that protons can pass through an intact $h$-BN layer, and that this process is facilitated by the presence of a Pt group metal (see Methods). In this scenario, the hydrated protons in the liquid electrolyte are likely stripped of their solvation shell the instant intercalation occurs, or a Grotthuss-type transport mechanism may be involved [22].

Stiction of the electrolyte on the $h$-BN surface is studied by measuring dynamic contact angles, which differ from the equilibrium value in Young's equation. This can be directly observed if a liquid drop on a solid loses volume by evaporation, where the drop footprint will start to move below a critical contact angle only (the receding angle). Likewise, when the volume of the drop is gradually increased, the footprint moves when the advancing contact angle is reached $[23,24]$. The difference in advancing and receding angles and the 
concomitant contact angle hysteresis are a macroscopic expression of adhesion and stiction. In Figure 3, we show dynamic contact angle measurements for applied potentials around the hydrogen adsorption peak. The angles as determined from inflating and deflating the drops in the corresponding videos (Supplementary Information, Videos 1,2) are shown for a potential above (Figure 3a) and below (Figure 3b) the hydrogen peak, i.e., for the corrugated and the flat surface, respectively. The drop volume was changed periodically and distinct receding angles $\theta_{\mathrm{r}}$, as well as advancing angles $\theta_{\mathrm{a}}$ were observed. Four positions are shown as photographs of the electrolyte drop on a $h$-BN/Rh(111) sample with the capillary, $0.85 \mathrm{~mm}$ in diameter, entering the drop from the top. The experiments show distinct hysteresis in the contact angles in the order of 60 degrees. The observed angles also depend on residual defects, which is reflected in the asymmetry between the left and the right footprint and sudden angle changes that are reproduced for subsequent cycles. All the effects due to residual imperfections, however, are distinct from the effect of the electrochemical potential. The size dependence of the wetting hysteresis is a consequence of the line tension, i.e., the work required for changing the length of the drop perimeter [25]. If the wetting angles are plotted vs. the inverse of the footprint radius (Figure 3c), the line tension and the extrapolation of the wetting angle for infinite footprints may be inferred. In Figure 3d, these extrapolated advancing and receding angles are plotted for a large set of experiments as a function of the applied potential, correlating the applied substrate potential with the measured contact angles. Clearly, the hystereses for potentials above $-200 \mathrm{mV}$ are different from those below this threshold of the hydrogen adsorption peak. Further experiments indicate that neither visible evolution of molecular hydrogen (where the reduction current increases exponentially in the voltammogram), nor electrowetting effects [26, 27] can be responsible for the observed changes in contact angle.

The concept for stiction and the concomitant contact angle hysteresis is sketched in Figure 4: the static friction $\sigma$ balances the forces that distort the Young equilibrium $\gamma_{S}-\gamma_{L S}-\gamma_{L} \cos \left(\theta_{0}\right)$ $=0$. The static friction $\sigma$ acts parallel to the surface as do $\gamma_{\mathrm{S}}$ and $\gamma_{\mathrm{LS}}$ (the tensions of the solidgas and the liquid-solid interface) and invokes a new equilibrium angle $\theta_{\sigma}$. The new equilibrium is determined by

$\gamma_{\mathrm{S}}-\gamma_{\mathrm{LS}}-\gamma_{\mathrm{L}} \cos \left(\theta_{\sigma}\right)+\sigma=0$ 
The static friction $\sigma$ may, however, not exceed a critical value $\pm \gamma_{\mathrm{H}}$, above which the footprint of the drop starts to move with an advancing or a receding angle. In Figure $3 c$ and $d$, the sizeindependent receding and advancing angles (i.e., the extrapolation to infinite footprints) $\theta_{\mathrm{r}}$ and $\theta_{\mathrm{a}}$ are indicated for the corrugated and the flat $h$-BN layer. Their values are given by

$\cos \left(\theta_{r, a}\right)=\cos \left(\theta_{0}\right) \mp \frac{\gamma_{H}}{\gamma_{L}}$

where $\gamma_{\mathrm{H}} \geq \sigma$ is the stiction threshold and $\gamma_{\mathrm{L}}$ is the gas-liquid interfacial energy or tension. With the knowledge of $\gamma_{\mathrm{L}}\left(72 \mathrm{~mJ} / \mathrm{m}^{2}\right.$ for water), which does not depend on the surface of the solid, and the determination of $\theta_{\mathrm{a}}$ and $\theta_{\mathrm{r}}$, the stiction threshold $\gamma_{\mathrm{H}}$ and the Young equilibrium angle $\theta_{0}$ may be directly determined.

Our experiments indicate that the hydrogen intercalation induced flattening of the $h$-BN nanomesh increases the Young wetting angle $\theta_{0}$ from $54.9 \pm 0.8$ to $64.5 \pm 1.7$ degrees. Using the Young-Dupré equation we determine the adhesion energy per unit area from $w_{\mathrm{LS}}=\gamma_{\mathrm{L}}+\gamma_{\mathrm{S}}-$ $\gamma_{\mathrm{LS}}=\gamma_{\mathrm{L}}\left(1+\cos \left(\theta_{0}\right)\right)$ from the Young equilibrium angle $\theta_{0}$ [28]. The adhesion energy decreases from $(1.58 \pm 0.01) \gamma_{\mathrm{L}}$ by $9.2 \pm 1.8 \%$ in going from the corrugated to the flat layer. The stiction threshold $\gamma_{\mathrm{H}}$ of $(0.33 \pm 0.01)$ and $(0.33 \pm 0.03) \gamma_{\mathrm{L}}$ for corrugated and flat is within the error bars the same for the two interfaces. The change in adsorption energy is incompatible with a Wenzel model [5], where an effective surface area change is responsible for a change in wetting angle: The effective surface area of the corrugated $h-\mathrm{BN}$ is $3 \%$ or $0.5 \%$ larger than that of the flat layer if the calculated corrugation of $316 \mathrm{pm}$ [29], or $83 \mathrm{pm}$ [17] are used. Apparently an electronic effect convoying the change of the corrugation must be at work in order to explain the magnitude of the effect. We argue that this is due to the loss of the lateral electric fields (2 $\mathrm{nm}$ dipole rings) [4] in the hydrogen-intercalated surface. The ratio of adsorption energies can be tested with a comparison to water adsorption [30]. Taking the calculated adsorption energies of water [31] for different adsorption sites within the $h$ $\mathrm{BN} / \mathrm{Rh}(111)$ supercell we calculate the average adsorption energies of the corrugated and the flat state. If we take for the corrugated case the portion of the wire-, pore- (hole) and rimregions from Xe-adsorption [4], and set the flat state equivalent to the unfavourable adsorption on the wires, we find an average adsorption energy that is $18 \%$ lower for water monomers and 9\% lower for hexamers on intercalated $h$-BN compared to the $h$-BN nanomesh. The comparison to the experiment is much better for hexamers, which makes sense since 
hexamers represent the simplest possible "drop" where only every second water molecule makes a hydrogen bond to the boron nitride [30]. Beyond these estimations of the adsorption energies involved in the switching phenomenon, the comprehensively characterised structure of the nanomesh makes this surface highly attractive for full scale theoretical treatments of wetting, which is difficult for less ordered systems. First molecular dynamics results estimate the change in adhesion (adsorption energy) at 4\% [6], which compares favourably with the $9.2 \%$ extracted from our wetting angle data; the quantitative theoretical description of stiction, on the other hand, is still far afield.

In summary, we have presented a switchable surface on which macroscopic static friction and adhesion can be linked to our microscopic understanding of water adsorption on this surface. The relatively simple structure of the $h$-BN nanomesh renders it amenable to accurate descriptions down to the atomic level, and an attractive model system for full-scale theoretical analyses of switchable surfaces, wetting, friction and lubrication. The fact that the intercalated surface survives emersion from the electrolyte may make this test system widely applicable for the study of friction and lubrication, also for adhering objects different from liquid drops. In biology we may think of controlled switching between high and low adhesiveness to cells, opening new avenues for studying cell migration towards the formation of complex multi-cellular arrangements. The high thermal and chemical stability opens technological opportunities, also in harsh environments, including influencing capillary action, stiction and adhesion in microfluidic or nanoelectromechanical devices. Finally, beyond the field of adhesion and friction, electrochemical hydrogen intercalation may find use in obtaining freestanding boron nitride layers, or more exotically, to stably confine tritium beneath a maximally transparent single atomic layer moderator as needed for the determination of the neutrino mass through beta-decay spectroscopy [32].

\section{References}

1. Persson, B. Sliding Friction (Eds. von Klitzing K., Wiesendanger, R.) Springer, 1999.

2. Wagner, T. J. W. \& Vella D. Switch on, switch off: stiction in nanoelectromechanical switches. Nanotechnology 24, 275501 (2013).

3. Corso, M. et al., Boron nitride nanomesh. Science 303, 217-220 (2004).

4. Dil, H. et al., Surface trapping of atoms and molecules with dipole rings. Science 319, 1824-1826 (2008).

5. Wenzel, R. N. Surface roughness and contact angle. J. Phys. Chem. 53, 1466-1467 (1949). 
6. Golze, D., Hutter, J. \& Iannuzzi, M. Wetting of water on hexagonal boron nitride@ $\mathrm{Rh}(111)$ : A QM/MM model based on atomic charges derived for nanostructured substrates. Phys. Chem. Chem. Phys. 17, 14307-14316 (2015).

7. Ternes, M, Lutz, C. P., Hirjibehedin, C. F., Giessibl, F. J. \& Heinrich, A. J. The force needed to move an atom on a surface. Science 319, 1066-1069 (2008).

8. Krim, J., Solina, D. H. \& Chiarello, R. Nanotribology of a Kr monolayer: a quartz crystal microbalance study of atomic-scale friction, Phys. Rev. Lett. 66, 181-184 (1991).

9. Ehrlich, G. \& Hudda, F. Atomic view of surface self-diffusion - tungsten on tungsten. $J$. Chem. Phys. 44, 1039-1049 (1966).

10. Evans, M. \& Polanyi, M. Inertia and driving force of chemical reactions. Trans. Faraday Soc. 34, 0011 (1938).

11. Zhang, Q., Qi, Y., Hector Jr., L. G., Cagin, T. \& Goddard III, W. A. Origin of static friction and its relationship to adhesion at the atomic scale. Phys. Rev. B 75, 144114 (2007).

12. Ichimura, K., Oh, S.-K. \& Nakagawa, M. Light-driven motion of liquids on a photoresponsive surface. Science 298, 1624-1626 (2002).

13. Sun, T. et al. Reversible switching between superhydrophilicity and superhydrophobicity. Angew. Chem., Int. Ed. 43, 357-360 (2004).

14. Lahann, J. et al. A reversibly switching surface. Science 299, 371-374 (2003).

15. Berner, S. et al., Boron nitride nanomesh: functionality from a corrugated monolayer. Angew. Chem., Int. Ed. 46, 5115-5119 (2007).

16. Widmer, R. et al., Electrolytic in situ STM investigation of h-BN-nanomesh. Electrochem. Commun. 9, 2484-2488 (2007).

17. Brugger, T. et al., Nanotexture switching of single-layer hexagonal boron nitride on rhodium by intercalation of hydrogen atoms. Angew. Chem., Int. Ed. 49, 6120-6124 (2010).

18. Willman, K. W. \& Murray, R. W. Contact angle between water and a poly(vinylferrocene) film on a potential-controlled platinum electrode. Anal. Chem. 55, 11391142 (1983).

19. Sung, Y. E., Thomas, S. \& Wieckowski, A. Characterization of the Rh(111) electrode by CEELS, AES, LEED, and voltammetry. Adsorption of (bi)sulfate, perchlorate, and carbon monoxide. J. Phys. Chem. 99, 13513-13521 (1995).

20. Colonell, J. I., Curtiss, T. J. \& Sibener, S. J. Coverage dependence of the kinetics for $\mathrm{H}_{2}$ desorption from Rh(111). Surface Sci. 366, 19-28 (1996).

21. $\mathrm{Hu}, \mathrm{S}$. et al. Proton transport through one-atom-thick crystals. Nature 516, 227-230 (2014). 
22. Marx, D., Tuckerman, M. E., Hutter, J. \& Parrinello, M. The nature of the hydrated excess proton in water. Nature 397, 601-604 (1999).

23. de Gennes, P. G. Wetting: statics and dynamics. Rev. Mod. Phys. 57, 827-863 (1985).

24. Kwok, D. Y. \& Neumann, A. W. Contact angle measurement and contact angle interpretation. Adv. Colloid Interface Sci. 81, 167-249 (1999).

25. Amirfazli, A., Hanig, S., Müller, A. \& Neumann, A. W. Measurements of line tension for solid-liquid-vapor systems using drop size dependence of contact angles and its correlation with solid-liquid interfacial tension. Langmuir 16, 2024-2031 (2000).

26. Schneemilch, M., Welters, W. J. J., Hayes, R. A. \& Ralston, J. Electrically induced changes in dynamic wettability. Langmuir 16, 2924-2927 (2000).

27. Manukyan, G., Oh, J. M., van den Ende, D., Lammertink, R. G. H. \& Mugele, F. Electrical switching of wetting states on superhydrophobic surfaces: a route towards reversible Cassie-to-Wenzel transitions. Phys. Rev. Lett. 106, 014501 (2011).

28. Schrader, M. E. Young-Dupré revisited. Langmuir 11, 3585-3589 (1995).

29. Iannuzzi, M. et al. Site-selective adsorption of phthalocyanine on h-BN/Rh(111) nanomesh. Phys. Chem. Chem. Phys. 16, 12374-12384 (2014).

30. Ma, H. et al. Chiral distortion of confined ice oligomers $(\mathrm{n}=5,6)$ Langmuir 28, 1524615250 (2012).

31. Ding, Y., Iannuzzi, M. \& Hutter, J. Investigation of boron nitride nanomesh interacting with water. J. Phys. Chem. C 115, 13685-13692 (2011).

32. Holzschuh, E., Fritschi, M. \& Kündig, W. Measurement of the electron neutrino mass from tritium $\beta$-decay. Phys. Lett. B 287, 381-388 (1992). 

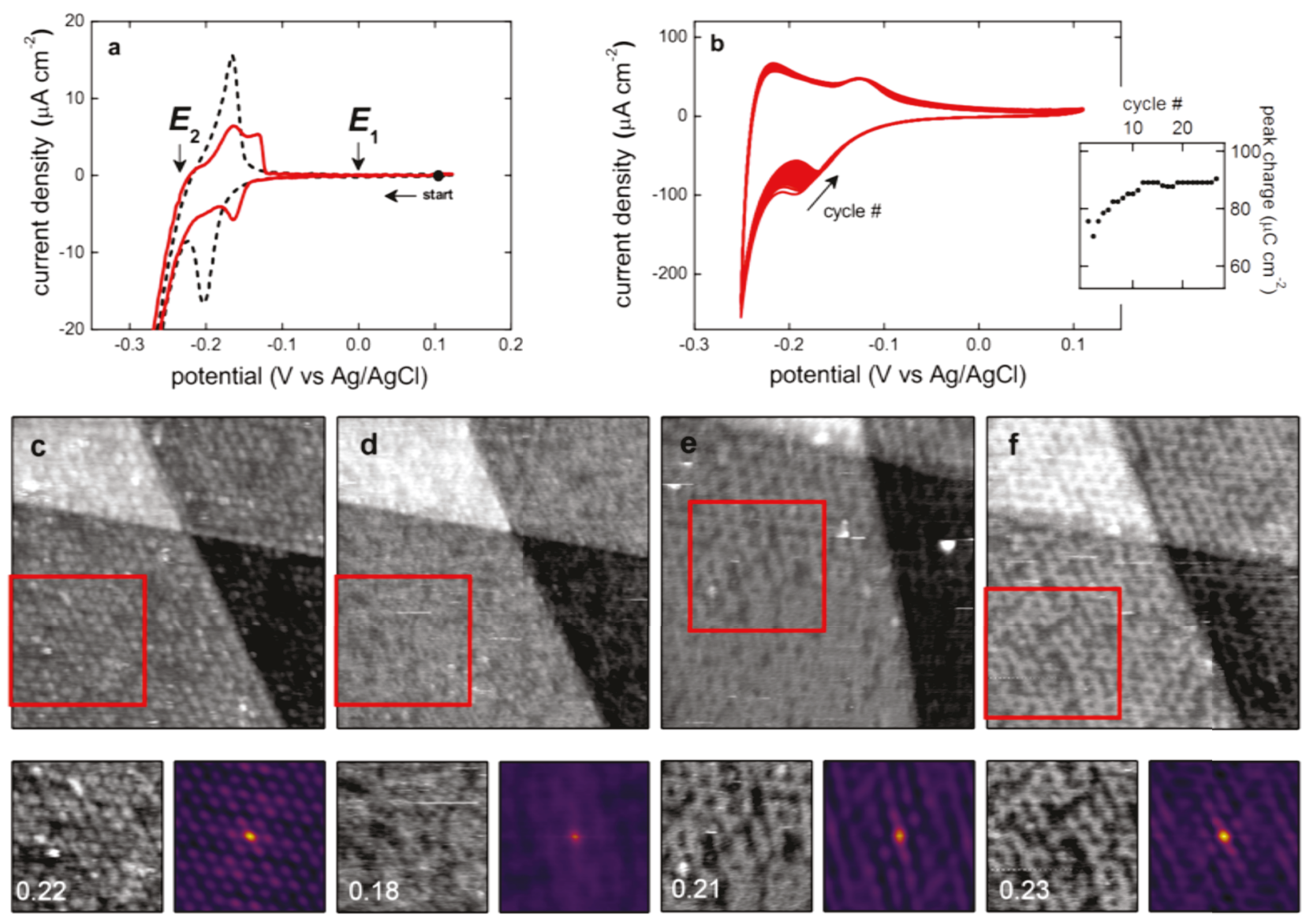

g
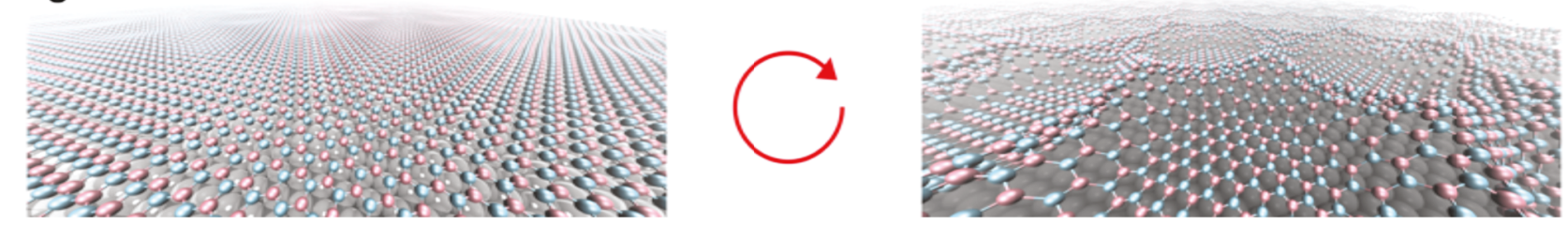

Figure 1. Voltammetry and electrochemical STM. (a) Cyclic voltammogram in a sessile electrolyte drop of h-BN nanomesh on $\mathrm{Rh}(111)$ (red trace) and of $\mathrm{Rh}(111)$ (black dashed trace) in $0.1 \mathrm{M} \mathrm{HClO}_{4}$. Scan rate $1 \mathrm{mV} \mathrm{s}^{-1}$. The hydrogen adsorption peaks are found at -200 and $-150 \mathrm{mV}$, respectively. (b) Stability of the hydrogen adsorption/desorption peaks on multiple cycling (28 cycles shown, recorded in a standard electrochemical cell. Scan rate 10 $\mathrm{mV} \mathrm{s}^{-1}$ ). The charge of the hydrogen adsorption peak as a function of cycle number is shown in the inset. (c-f) Sequence of in situ STM images at various substrate potentials for a region with three atomic terrace levels. The red square is the same sample area, and is shown as zoom-ins. The white numbers are the RMS roughness in units of the terrace height. The colour squares are the autocorrelations of these zoom-ins and reveal, if present, the $3.2 \mathrm{~nm}$ periodicity of the $h$-BN superstructure. (c) $E_{1}=0 \mathrm{~V}$; (d) $E_{2}=-0.25 \mathrm{~V}$; (e) switching $E_{2} \rightarrow E_{1}$ (image scanned from bottom to top); (f) $E_{1}$ after recovery of corrugation. Image sizes $66 \times 66$ $\mathrm{nm}^{2}$, tunnelling current $0.1 \mathrm{nA}$, tip potential fixed at $-0.45 \mathrm{~V}$. (g) 3-dimensional representation of the flat (left) and the corrugated h-BN layer (right). N: sky blue, B: pink, $\mathrm{Rh}$ : dark grey, $\mathrm{H}$ : white. For clarity, the heights of $\mathrm{N}$ and $\mathrm{B}$ above the $\mathrm{Rh}$ top layer have been stretched by a factor of three. Coordinates taken from [17] and [29]. 


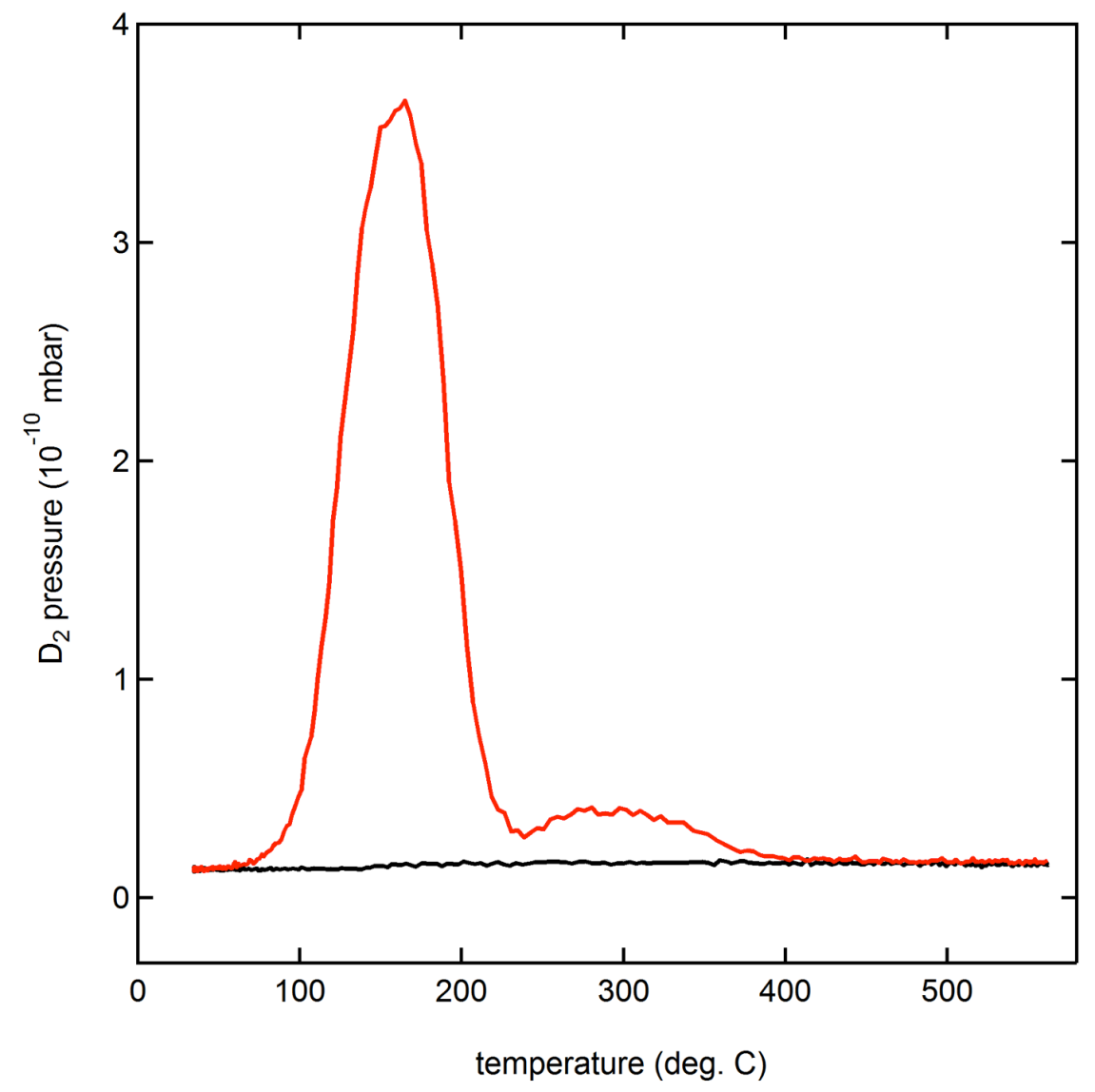

Figure 2. Deuterium thermal desorption spectra (TDS). $h$-BN/Rh(111) was exposed to $0.1 \mathrm{M} \mathrm{DClO}_{4}$ in $\mathrm{D}_{2} \mathrm{O}$ at substrate potentials $E_{1}=0 \mathrm{~V}$ (black trace) and $E_{2}=-0.25 \mathrm{~V}$ (red trace). After loading the samples in the electrolyte they were transferred to ultrahigh vacuum for TDS (see Methods). Heating rate $0.9 \mathrm{~K} \mathrm{~s}^{-1}$. 

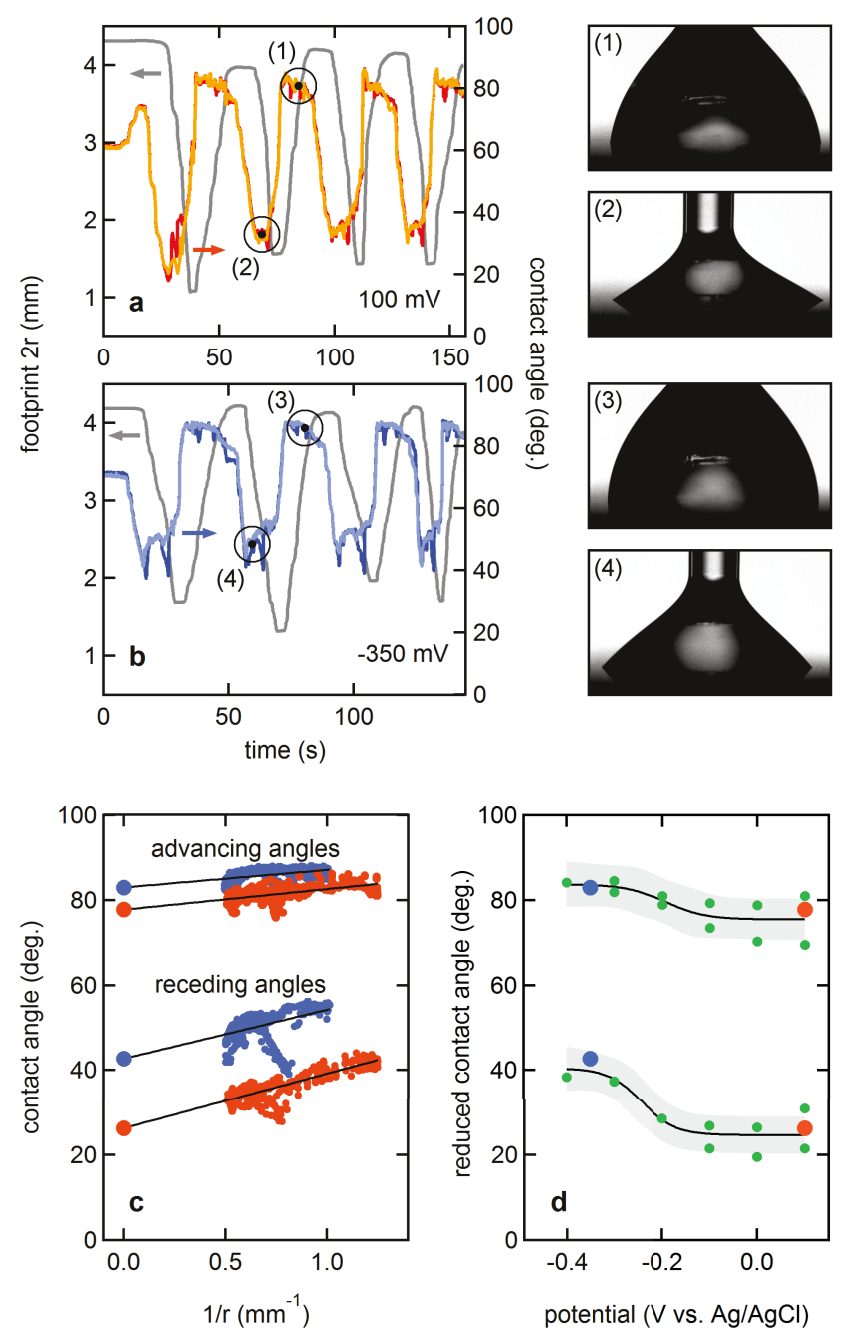

Figure 3. Dynamic contact angle measurements. (a) Left (orange) and right (red) contact angles and drop footprints (diameters $2 r$ ) as function of time. Two pictures for an advancing and a receding drop as indicated in the transients at $100 \mathrm{mV}$ (corrugated state). (b) Left (blue) and right (turquoise) contact angles and footprints as function of time. Two pictures as indicated in the transients at $-350 \mathrm{mV}$ (flat state). (c) Contact angles of (a) and (b) versus $1 / \mathrm{r}$ for times above 40 seconds. Extrapolation to zero yields reduced contact angles independent of the contact line. (d) Reduced receding and advancing angles for different applied potentials, where the hydrogen intercalation peak is found at about $-0.2 \mathrm{~V}$. The full circles indicate the values from the series in (a) and (b). The solid lines are sigmoid fits from which the corresponding angles for all experiments are determined. Values of $\theta_{\mathrm{a}} ; \theta_{\mathrm{r}}\left(76.0 \pm 1.2^{\circ}\right.$; $\left.24.5 \pm 1.4^{\circ}\right)$ for the corrugated and $\left(84.3 \pm 2.3^{\circ} ; 40.1 \pm 3.2^{\circ}\right)$ for the flat state are found. Sigmoid fits to all experimental angles as shown. The grey band indicates the $1 \sigma$ prediction interval. 
advancing

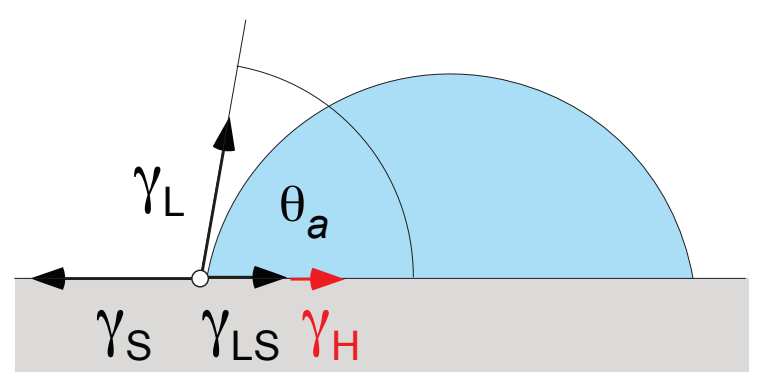

receding

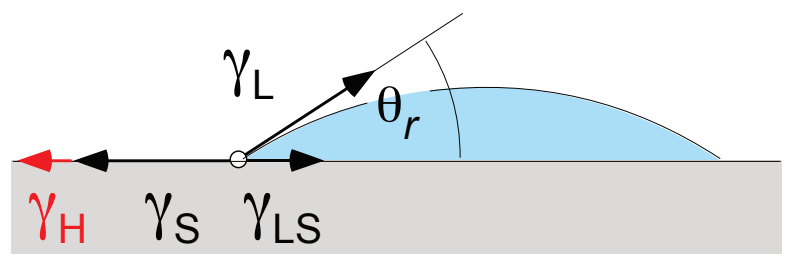

Figure 4. Wetting angle hysteresis and stiction. The footprint of a drop starts to move with an advancing (left panel) or a receding (right panel) angle $\theta_{\mathrm{a}}$ or $\theta_{\mathrm{r}}$, respectively, if the extra lateral tension exceeds the stiction threshold $\gamma_{\mathrm{H}}$. The equilibrium angle from Young's equation $\theta_{0}$ lies between $\theta_{\mathrm{a}}$ and $\theta_{\mathrm{r}} . \gamma_{\mathrm{S}}, \gamma_{\mathrm{LS}}$ and $\gamma_{\mathrm{L}}$ are the corresponding interface energies solid-gas (S), liquid-solid (LS) and liquid-gas (L).

\section{Acknowledgements}

Financial support by the Swiss National Science Foundation within the funding instrument "Sinergia" is gratefully acknowledged. S.F.L.M. acknowledges the receipt of an FP7 Marie Curie European reintegration grant, ERC grant OxideSurfaces and together with S.D.F. support by FWO-Vlaanderen. We thank Michael Schmid for help with the depiction of the STM images, Gerhard Schütz for discussions and Ari Pavo Seitsonen for the artwork in Figure 1g.

\section{Author statements}

T.G. conceived the project together with S.F.L.M., who designed and performed the electrochemical experiments, in situ STM and in situ contact angle measurements. A.H. prepared the nanomesh samples, analysed contact angle data and performed the TDS measurements. S.M. built the contact angle apparatus. O.G. drew our attention to dynamic contact angle measurements. S.D.F. and J.O. managed the Sinergia project. All authors contributed to discussions. S.F.L.M., A.H. and T.G. prepared the manuscript.

Reprints and permissions information is available at www.nature.com/reprints. The authors declare no competing financial interests. Readers are welcome to comment on the online version of the paper. Correspondence and requests for materials should be addressed to S.F.L.M. (stmerten@gmail.com, ORCID 0000-0002-5715-0486) or T.G. (greber@physik.uzh.ch). 


\section{Methods}

Experimental details. The experiments were performed in a clean room on single layer h-BN grown on $150 \mathrm{~nm}$ thick single crystalline rhodium films on $\mathrm{Si}(111)$ wafers which were protected with a $40 \mathrm{~nm}$ yttria stabilised zirconia (YSZ) diffusion barrier [33]. The wetting angles were determined by a lab-built CCD camera set up with 15 frames per second and an automated angle evaluation scheme (see Error analysis). Electrochemical potential control was achieved using a Metrohm-Autolab PGSTAT101 potentiostat, using a lab-built glass syringe carrying a $\mathrm{Ag} / \mathrm{AgCl} / 3 \mathrm{M} \mathrm{NaCl}$ reference and $\mathrm{Pt}$ wire counter electrode. All electrochemical potentials are reported versus the $\mathrm{Ag} / \mathrm{AgCl}$ reference. All glassware, including the glass capillary for in situ contact angle measurements, was cleaned by boiling in $20 \%$ nitric acid and rinsing with ultrapure water (MilliQ, Millipore, $18.2 \mathrm{MOhm} \mathrm{cm}$ ), to ensure perfect wetting. The electrolyte was prepared from reagent grade $70 \% \mathrm{HClO}_{4}(\mathrm{Sigma}-$ Aldrich) and ultrapure water. For the deuterium desorption experiments, light water was replaced with $\mathrm{D}_{2} \mathrm{O}$ (Fluka, $\mathrm{D}>99.8 \%$ ). All operations were carried out in an Ar filled glove box in a clean room [33]. In situ STM was performed with an Agilent PicoLE system, using an electrochemically etched W tip which was coated with a thermoplastic polymer to minimise the Faradaic current. The thermal desorption experiments were performed in an apparatus equipped with a calibrated quadrupole mass spectrometer (QMS 200 M2, Pfeiffer Vacuum), and pumped with a turbomolecular pump (TMU 071, Pfeiffer Vacuum) [17].

Thermal and chemical stability of the $\boldsymbol{h}$-BN/Rh nanomesh. Cumulative experience in the authors' laboratories since the discovery of the boron nitride nanomesh [3] indicates a shelf life of several years under ambient conditions, stability on exposure to and ultrasonication in common solvents (both polar and nonpolar), and after 15-min cycles in commercial UVozone cleaners. Furthermore, the nanomesh can be heated to $400{ }^{\circ} \mathrm{C}$ in air and close to $1000{ }^{\circ} \mathrm{C}$ in vacuum [4]. In contrast, when exposed to hot strongly alkaline solutions, the $h$ $\mathrm{BN}$ appears to be removed, in line with observations for bulk $h$-BN [34]. Low-energy $\mathrm{Ar}^{+}$ bombardment followed by annealing has been shown to produce atomically precise defects [35]. Hydrogen intercalation appears not to adversely affect the stability of the boron nitride layer [17]. As shown here, electrochemical cycling can be performed many times without signs of deterioration (Fig. 1b). Emersion of the intercalated sample and transfer to vacuum are possible and yield similar amounts of intercalated hydrogen as in an all-vacuum experiment (next section, main text Fig. 2 and [17]). 
Adventitious adsorption from solution or the laboratory atmosphere was not observed, and attempts to adsorb organic molecules from solution were unsuccessful. These observations, together with the widely available cleaning methods listed above, make the nanomesh amenable to experiments under standard laboratory conditions.

Ambient-to-UHV transfer and TDS. A critical factor in our ambient-to-vacuum experiment is the successful emersion of the sample from the electrochemical environment, which means that potential control is required until the solid-bulk electrolyte interface ceases to exist. This way, the $h$-BN/Rh-electrolyte system is never allowed to assume its open circuit potential, where electrochemical deintercalation of the hydrogen may occur. This also explains why we can rinse the sample with ultrapure water to remove superficially adsorbed deuterium and traces of perchloric acid, before transferring the sample to vacuum. The successful extraction of the sample from the electrolyte is aided by its mild intrinsic hydrophobicity, much akin to the pioneering work by Hansen and Kolb [36], who studied hydrophobic adlayers on noble metal electrodes after transfer to vacuum.

The sample mounting and transfer into the vacuum chamber was a process of about 10 min, during which the sample was exposed to water and ambient air. Because we expected that the remaining solvent molecules on the sample may still contribute to a deuterium signal during the desorption experiment, the sample was rinsed with ultrapure water after removal from the $\mathrm{D}_{2} \mathrm{O}$ solution and before entry in the vacuum system. Artifacts from this procedure were excluded by comparing TDS spectra from two samples that were handled identically, but of which only one was held at the potential required for intercalation (see Fig. 2 of the main text).

In the design of the sample holder, special precautions were taken to limit heating and desorption as much as possible to the sample itself, and avoid a background covering the deuterium signal from the surface or even risking the chamber pressure to exceed the safe operation limit of the mass spectrometer channel electron multiplier. In order to maximise the signal-to-noise ratio, heating should occur as locally and as fast as possible. Extended Data Fig. 1(a) shows the sample holders used, which allow the sample to be annealed via resistive heating through the 100-nm thick Rh film [33] solely. In this configuration, only the top tungsten clamps became as hot as the sample and temperatures of $580{ }^{\circ} \mathrm{C}$ could be reached with a maximum pressure load of about $1.2 \times 10^{-6} \mathrm{mbar}$. The sample configuration requires the 
use of a pyrometer to assess the sample temperature, which was calibrated against a thermocouple reading of a Rh sample as shown in Extended Data Fig. 1(b). As the lowest measureable temperature by the pyrometer is around $350{ }^{\circ} \mathrm{C}$, additional extrapolation of the curve sections outside this region was required.

Extended Data Fig. 1(b) displays all relevant information of a desorption experiment, such as the applied power to the sample, pyrometer, mass spectrometer and integral pressure readings. To determine the sample temperature, the following differential equations were used, which consider the power applied to the sample and its heat loss through radiation and thermal conduction to the sample holder:

$$
\begin{aligned}
& \frac{d T}{d t}=a \times P(t)+b \times P(t) \times T(t) \\
& \frac{d T}{d t}=a \times P(t)+b \times T(t)
\end{aligned}
$$

with applied power $P$, sample temperature $T$, time $t$ and fitting parameters $a$ and $b$. Best results were obtained using Eq. (M1) ascending temperature region and Eq. (M2) for the descending temperature region of the heating/cooling curve. The extrapolated temperature was finally a complete numerical simulation of the temperature evolution, with room temperature as the starting point and the applied power being the only input variables.

Hydrogen (de)intercalation rate. The hydrogen intercalation we study is closely related to underpotential deposition of hydrogen $(\mathrm{H}$ upd), i.e. electrosorption at potentials positive from the hydrogen evolution equilibrium potential, which is known to occur on the platinum group metals [37], and to involve comparable energies on Pt and $\mathrm{Rh}[38,39]$. As shown in Figure 1a, hydrogen intercalation into $h$-BN/Rh occurs at a potential $c a .50 \mathrm{mV}$ less negative than does $\mathrm{H}$ upd on bare $\mathrm{Rh}(111)$, which may be due to the fact that intercalating $\mathrm{H}$ does not have to compete with $\mathrm{ClO}_{4}^{-}$for adsorption on $\mathrm{Rh}$ after passing the $\mathrm{BN}$ overlayer [19].

From the in situ STM image in Figure 1e, recorded immediately after switching the substrate potential from a value where intercalated hydrogen is stable to a value where electrochemical deintercalation takes place, it is apparent that recovery of the corrugation requires about half 
of the time to record one image. If the local corrugation is taken as a measure of deintercalation, the line speed $\left(8.1\right.$ lines s$\left.^{-1}\right)$ and image resolution $(512 \times 512$ pixels $)$ point at a characteristic time of $\approx 30 \mathrm{~s}$. The forward process (intercalation) reproducibly led to a flattening in EC-STM in $<1 \mathrm{~s}$ (Extended Data Fig. 2), indicating that intercalation is considerably faster than deintercalation. Restoration of the corrugation must be considered as the last step in the electrochemical deintercalation; it is not obvious which is the ratedetermining process, to which the pore and wire areas of the nanomesh may contribute differently. The observed difference in intercalation and deintercalation rate may originate from unilateral catalytic activation by the Rh substrate [21], i.e. the different proton affinity of the $h$-BN/Rh and the $h$-BN/electrolyte interface and is also convoluted with the kinetics of $\mathrm{H}$ upd [40].

Error analysis. The determination of the contact angles from one image frame is based on 32 sigmoid function fits to the optical contrast change for the left and right periphery of the drop, in order to determine the location of the liquid drop edge. These fits, based on 60 raw data pixels, are all located in an interval of $0.25 \mathrm{~mm}$ centred at the footprint of the drop and yield a fitting error for the edge location in the order of $0.8 \mu \mathrm{m}$. This is 5 times less than the optical pixel width of the image and can be explained by the very sharp optical contrast between background and liquid drop. The angle is then fitted with two separate linear functions, one for the real edge between background and drop and one for its mirror image. The crossing of these two lines reveals the location of the footprint and the contact angle was half the angle between the two fitted lines. Eq. (M3) is valid for the left and right angles, where $\alpha$ is the contact angle and $b_{1}$ and $b_{2}$ are the slopes of the two linear fits (real angle and mirror angle).

$$
\begin{aligned}
& \alpha=0.5 \times\left(\arctan \left(a b s\left(b_{1}\right)\right)+\arctan \left(a b s\left(b_{2}\right)\right)\right. \\
& \Delta \alpha=\sqrt{\left(\frac{0.5}{1+b_{1}{ }^{2}} \sigma b_{1}\right)^{2}+\left(\frac{0.5}{1+b_{2}{ }^{2}} \sigma b_{2}\right)^{2}}
\end{aligned}
$$

Gaussian error propagation of Eq. (M3) yields Eq. (M4) where $\sigma b_{i}$ is the error of the slope fits and the accuracy of one measured contact angle is determined to be about $0.3^{\circ}$. For each extrapolation of the wetting angles to $1 / \mathrm{r}=0$ we used about 400 angles, which were determined with the procedure above. The four linear fits shown in Figure 3C are based on 
412, 410, 344 and 402 individual angle data points, respectively. Because of the high accuracy and the large number of data, we may neglect the error of the individual angles. Visibly larger is the influence of e.g. residual defects, which induce asymmetries between the left and right angle and angle spikes (Figure 3a-c). The error of the reduced contact angle is the fitting error of the $\theta$-axis intersection and the largest error for the data sets in Figure $3 \mathrm{c}$ is $0.8^{\circ}$, which is well below the size of the printed dot.

In Figure $3 \mathrm{~d}$ we show all experiments and the corresponding advancing and receding angles performed on 3 different samples. The largest errors are not from the angle determination procedure described above, but mainly from differences of the samples, sample handling and manual operation of the syringe. The uncertainties that we cite are derived from the standard deviations of the mean values of the advancing and receding angles in Figure $3 \mathrm{~d}$, where advancing and receding angles are taken from the sigmoid fits at the same applied potential. Values of $\theta_{\mathrm{a}} ; \theta_{\mathrm{r}}\left(76.0 \pm 1.2^{\circ} ; 24.5 \pm 1.4^{\circ}\right)$ for the corrugated and $\left(84.3 \pm 2.3^{\circ} ; 40.1 \pm 3.2\right)$ for the flat state are found. The values and error bars for $\theta_{0}, \mathrm{w}_{\mathrm{LS}}$ and $\gamma_{\mathrm{L}}$ are determined from the four angles and their standard deviation with Gaussian error propagation.

33. Hemmi, A. et al. High quality single atomic layer deposition of hexagonal boron nitride on single crystalline Rh(111) four-inch wafers. Rev. Sci. Instr. 85, 035101 (2014).

34. King, S. W., Nemanich, R. J. \& Davis, R. F. Cleaning of pyrolytic hexagonal boron nitride surfaces. Surf. Interface Anal. 47, 798-803 (2015).

35. Cun, H., Iannuzzi, M., Hemmi, A., Osterwalder, J. \& Greber, T. Two-nanometer voids in single-layer hexagonal boron nitride: formation via the "can-opener" effect and annihilation by self-healing. ACS Nano 8, 7423-7431 (2014).

36. Hansen, W. N. \& Kolb, D. M. The work function of emersed electrodes. J. Electroanal. Chem. 100, 493-500 (1979).

37. Koper, M. T. M. Blank voltammetry of hexagonal surfaces of Pt-group metal electrodes: Comparison to density functional theory calculations and ultra-high vacuum experiments on water dissociation. Electrochim. Acta 56, 10645-10651 (2011).

38. Jerkiewicz, G. \& Zolfaghari, A. Determination of the energy of the metal-underpotentialdeposited hydrogen bond for rhodium electrodes. J. Phys. Chem. 100, 8454-8461 (1996).

39. Zolfaghari, A., Chayer, M. \& Jerkiewicz, G. Energetics of the underpotential deposition of hydrogen on platinum electrodes I. Absence of coadsorbed species. J. Electrochem. Soc. 144, 3034-3041 (1997).

40. Łosiewicza, B., Jurczakowski, R., Lasia, A. Kinetics of hydrogen underpotential deposition at polycrystalline rhodium in acidic solutions. Electrochim. Acta 56, 5746-5753 (2011). 

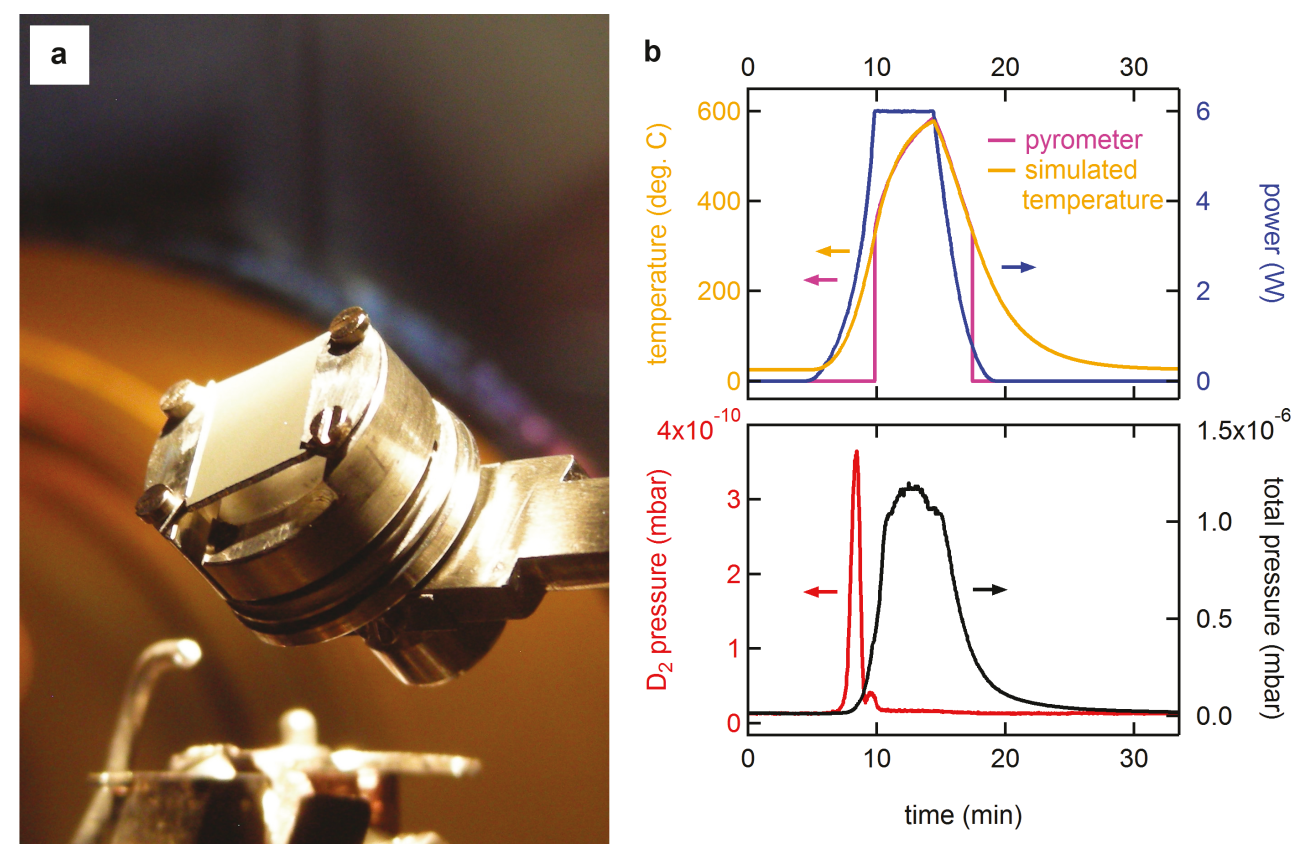

Extended Data Figure 1 - Thermal desorption spectroscopy. (a) h-BN/Rh(111) thin film sample and sample holder ready for the desorption experiment in the UHV chamber. (b) Thermal desorption experiment with temperature and power data in the upper part and pressure data in the lower part. The purple data are pyrometer readings, which were calibrated to a previous thermocouple measurement using a $\mathrm{Rh}$ crystal. The simulated temperature is a solution of Eq. M1 and M2 with the applied power and a starting temperature of $25{ }^{\circ} \mathrm{C}$ as input variables.

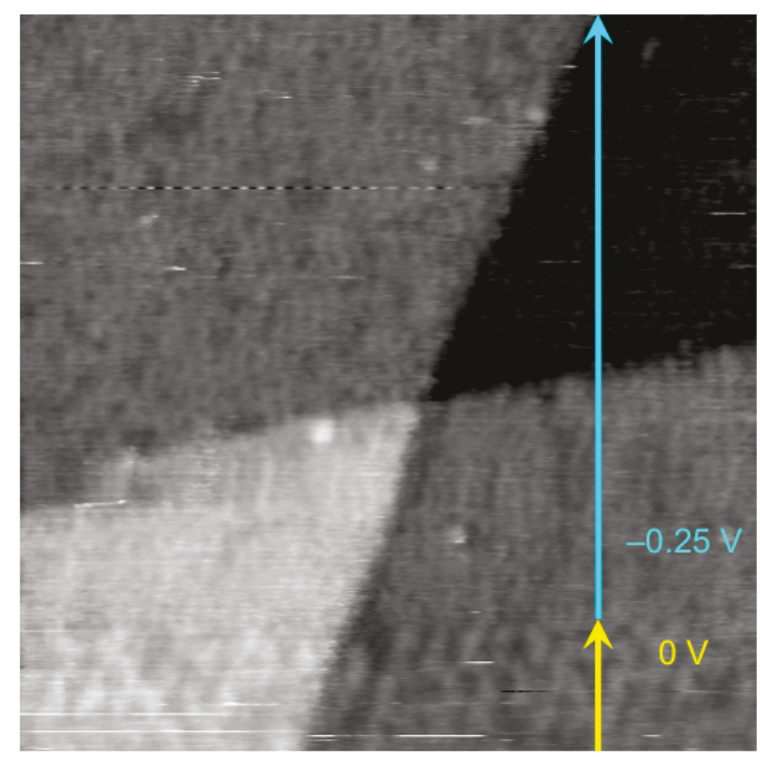

Extended Data Figure 2 - Electrochemical STM during hydrogen intercalation. The substrate potential during scanning (image scanned from bottom to top) was switched from $E_{1}$ $=0 \mathrm{~V}$ (deintercalated, yellow arrow) to $E_{2}=-0.25 \mathrm{~V}$ (intercalated, light blue arrow) at ca. one-fifth from the lower edge of the STM image. On the time scale of imaging, intercalationinduced flattening of the surface is instantaneous, in sharp contrast with deintercalation (compare main text Fig. 1e). Image sizes $66 \times 66 \mathrm{~nm}^{2}$, tunnelling current $0.1 \mathrm{nA}$, tip potential fixed at $-0.45 \mathrm{~V}$. 
Supplementary Information Guide for

\section{Switching stiction and adhesion of a liquid on a solid}

Videos S1 and S2

Video S1 (file Nanomesh_corrugated.mov)

Advancing, followed by receding electrolyte drop on $h-\mathrm{BN} / \mathrm{Rh}(111)$, at an applied substrate potential $E=+0.1 \mathrm{~V}$, where the nanomesh occurs in its normal corrugated state. The video shows all images captured between $t=68 \mathrm{~s}$ and $t=80 \mathrm{~s}$ in Figure $3 \mathrm{a}$.

Video S2 (file Nanomesh_flat.mov)

Advancing, followed by receding electrolyte drop on $h$-BN/Rh(111), at an applied substrate potential $E=-0.35 \mathrm{~V}$, where hydrogen intercalation has caused flattening of the nanomesh. The video shows all images captured between between $t=95 \mathrm{~s}$ and $t=115 \mathrm{~s}$ in Figure $3 \mathrm{~b}$. 